\title{
Impurity-induced tuning of quantum well states in spin-dependent resonant tunneling
}

\author{
Alan Kalitsov ${ }^{1}$ A. Coho, ${ }^{1}$ Nicholas Kioussis, ${ }^{1, \text { f }}$ Anatoly Vedyayev ${ }^{2}$ M. Chshiev ${ }^{3}$ and A. Granovsky ${ }^{2}$ \\ ${ }^{1}$ Department of Physics, California State University, Northridge, CA 91330-8268 \\ ${ }^{2}$ Faculty of Physics, Lomonosov Moscow State University, 119992 Moscow, Russia \\ ${ }^{3}$ Department of Physics, Virginia Polytechnic Institute and State University, Blacksburg, VA 24061-0435
}

(Dated: July 7, 2018)

\begin{abstract}
We report exact model calculations of the spin-dependent tunneling in double magnetic tunnel junctions in the presence of impurities in the well. We show that the impurity can tune selectively the spin channels giving rise to a wide variety of interesting and novel transport phenomena. The tunneling magnetoresistance, the spin polarization and the local current can be dramatically enhanced or suppressed by impurities. The underlying mechanism is the impurity-induced shift of the quantum well states (QWS) which depends on the impurity potential, impurity position and the symmetry of the QWS.
\end{abstract}

PACS numbers: 72.10.-d, 72.25.-b, 73.40.Gk

Tunneling of spin-polarized electrons through magnetic tunnel junctions (MTJ) has attracted [1] wide and sustained interest in the past few years, both experimentally $[2,[3,4,[5,[6]$ and theoretically $[3,7,6,8,9]$. This is due to the potential applications of MTJ in spin-electronic devices, such as magnetic sensors and magnetic randomaccess memories. The key point for these applications is the tunnel magnetoresistance (TMR), i.e. the dependence of the tunneling current on the relative orientation of the magnetization of the ferromagnetic layers, which can be changed by an applied magnetic field $[\underline{6}]$.

Double magnetic tunnel junctions (DMTJ) consist of a central metallic layer (quantum well) between two insulating barriers and two ferromagnetic electrodes. The insulating layers are thin enough for electrons to tunnel through the barriers if a bias voltage is applied between the electrodes. The TMR behavior in DMTJ is determined by quantum well states (QWS) formed in the middle layer when a resonance condition is fulfilled [5]. The TMR can be dramatically enhanced when spinpolarized electrons resonantly tunnel through the middle layer [7]. Theoretical formulations of the TMR in DMTJ are usually based on models which assume perfect systems [7, 9, 10, 11, 12, 13, 14]. The TMR exhibits an amplitude-varying oscillatory behavior as a function of the thickness of the middle layer with a period of $\pi / k_{F}^{\sigma}$, where $k_{F}^{\sigma}$ is the spin-dependent Fermi wave vector in the middle layer [10]. Chshiev et al introduced [9] phenomenologically the electron scattering in the middle layer without taking into account vertex corrections. Consequently, they introduced an effective electric field within each barrier in order to satisfy the continuity equation for the current [15].

However, actual MTJ contain large amounts of disorder in the electrodes, in the barriers, in the quantum well, and at the electrode/barrier or electrode/quantum well interfaces [3, 16]. This disorder may represent interdif-

*E-mail me at: nick.kioussis@csun.edu. fusion at the interfaces, interface roughness, and impurities. Experiments in single MTJ have suggested that disorder can affect the TMR in a critical way giving rise to impurity-assisted tunneling [3, 16]. While the effect of impurities within a single barrier has been studied theoretically recently [17, 18], its role in DMTJ remains an unexplored area thus far.

In this paper we present exact model calculations of the spin-dependent resonant tunneling in double MTJ structures to study for the first time the effect of magnetic and nonmagnetic impurities in the magnetic middle layer on the TMR, spin polarization, and local current as a function of external bias. This approach conserves the continuity of the current in contrast to Ref. [9]. We show that the impurities may induce a shift of the original QWS depending on the sign of the impurity scattering potential, the impurity position, and the symmetry of the original QWS. These effects can tune selectively the spin channels giving rise to a wide variety of novel and interesting spin-dependent transport phenomena, such as a dramatic enhancement or suppression of the TMR and the spin polarization, and a sign reversal of the spin polarization. Interestingly, the calculations reveal that even though the effect of the impurity on the average spin current is small for antisymmetric QWS, the local spin current exhibits strong variation.

We employ the free-electron band model to describe the electron tunneling in a double MTJ with impurities in the metallic middle layer of width $b$, whose scattering is modeled by a spin-dependent $\delta$-function scattering potential. Figure 1 shows a schematic diagram of the energy bands for the parallel configuration under a small external bias for the majority and minority spin carriers, respectively. The geometric parameters of the DMTJ are chosen in such a way that the spin-dependent energies of the QWS, $E_{R}^{\sigma}$, fall within the majority (minority) spin band and they are of antisymmetric (symmetric) character, respectively. The impurity potential $V^{\sigma}$ relative to the bottom of the band in the middle layer is also shown with green (red) line for positive (negative) sign, with the impurity placed at the center of the well. 

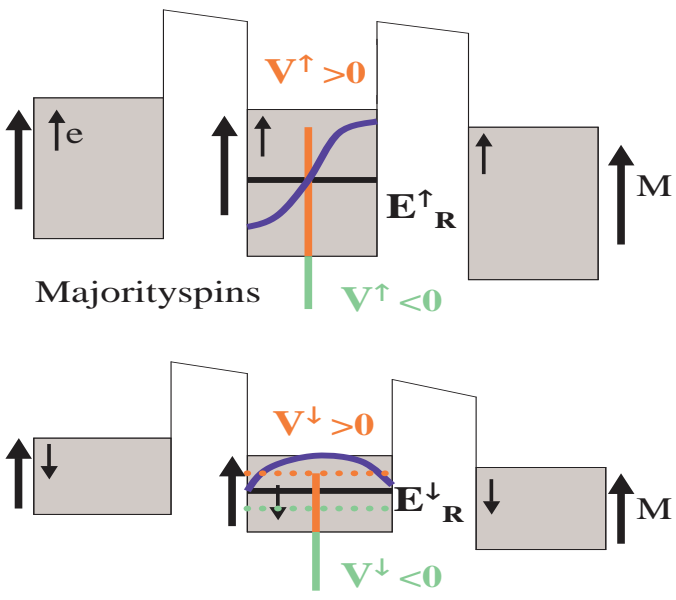

Minority spins

FIG. 1: Schematic energy diagram for the parallel configuration for the majority and minority spin carriers. The energies of the QWS denoted by $E_{R}^{\sigma}$, fall within the majority (minority) band and they are antisymmetric (symmetric), respectively. The impurity $\delta$-type potential $V^{\sigma}$ of positive (negative) sign relative to the bottom of the band in the well is denoted with red (green) line, respectively. There is no shift of the antisymmetric QWS, whereas the symmetric QWS shift towards higher (lower) values if $V^{\sigma}>0(<0)$.

Employing the WKB approximation in the barrier region, the one-electron Green's functions for the clean DMTJ are solutions of the Schrödinger equation,

$$
\begin{aligned}
& \left(E+\frac{\hbar^{2}}{2 m_{i}}\left(\frac{\partial^{2}}{\partial z^{2}}-\kappa^{2}\right)-\tilde{V}_{i}^{\sigma}-e v(z)\right) G_{\kappa}^{\sigma}\left(z, z^{\prime}\right)= \\
& =\delta\left(z-z^{\prime}\right)
\end{aligned}
$$

where $\tilde{V}_{i}^{\sigma}=V_{i}^{\sigma}$ in the metallic layers, and $\tilde{V}_{i}^{\sigma}=U_{i}$ in the barriers. Here, $V_{i}^{\sigma}$ is the spin-dependent potential (or the bottom of the band) of the $i$-th metal, $U_{i}$ is the potential barrier height in the ith barrier, and $v_{i}(z)$ is the voltage drop within the ith layer, assumed to be nonzero only in the barriers. The $z$ is the coordinate perpendicular to the interface, $\kappa$ is the in-plane wave vector of energy $E$, and $m_{i}$ is the electron effective mass in the $i$-th layer. The coefficients of the wave functions in each layer are determined by the boundary conditions at the interfaces.

The one-electron Green's function in the presence of the impurity is determined from the Dyson equation:

$$
\begin{aligned}
\tilde{G}^{\sigma}\left(\rho, z, \rho^{\prime}, z^{\prime}\right) & =G^{\sigma}\left(\rho, z, \rho^{\prime}, z^{\prime}\right)+G^{\sigma}\left(\rho, z, \rho_{0}, z_{0}\right) T^{\sigma} \times \\
& \times G^{\sigma}\left(\rho_{0}, z_{0}, \rho^{\prime}, z^{\prime}\right),
\end{aligned}
$$

where $T^{\sigma}=V^{\sigma}\left[1-V^{\sigma} G_{33}^{\sigma}\left(\rho_{0}, z_{0}, \rho_{0}, z_{0}\right)\right]^{-1}$ is the Tmatrix, $V^{\sigma}=V_{i m p}^{\sigma} \delta\left(z-z_{0}\right)-V_{3}^{\sigma}$, and $\rho_{0}, z_{0}$ is the impurity position. Here, $V^{\sigma}\left(V_{i m p}^{\sigma}\right)$ refers to the impurity potential relative to the bottom of the band (Fermi energy) of the well. Depending on the width of the quantum well and the sign of $V^{\sigma}$, the poles of the T-matrix give rise to impurity-induced shift of resonances, which can in turn enhance or suppress selectively the current density of the majority or minority spin channel for a given magnetization orientation.

The local current density for spin $\sigma$ is given by 19

$$
\begin{aligned}
j^{\sigma}\left(\rho-\rho_{0}, z\right) & =\frac{e}{\pi \hbar} \int\left[f(E)-f\left(E+e V_{\text {ext }}\right)\right] \times \\
& \times D^{\sigma}\left(E, \rho-\rho_{0}, z\right) d E
\end{aligned}
$$

where $f(E)$ is the Fermi-Dirac distribution function and the transmission probability $D^{\sigma}$ is 20 .

$$
D^{\sigma}\left(E, \rho-\rho_{0}, z\right)=\left(\frac{\hbar^{2}}{2 m}\right)^{2} \sum_{k, k^{\prime}} A_{k, k \prime}^{\sigma}, \overleftrightarrow{\nabla}_{z} \overleftrightarrow{\nabla}_{z^{\prime}} A_{k, k \prime}^{\sigma}
$$

Here, $\stackrel{\leftrightarrow}{\nabla}_{z}=(1 / 2)\left(\vec{\nabla}_{z}-\overleftarrow{\nabla}_{z}\right)$ is the antisymmetric gradient operator, $A_{k, k^{\prime}}^{\sigma}=(1 / 2)\left[G_{k, k^{\prime}}^{r e t, \sigma}-G_{k, k \prime}^{a d v, \sigma}\right]$, where $G_{k, k \prime}^{r e t, \sigma}$ and $G_{k, k,}^{a d v, \sigma}$ are the retarded and advanced Green's functions, respectively. The total transmission probability $D=D^{(0)}+D^{(1)}+D^{(2)}$, where $D^{(0)}$ is the transmission probability in the absence of impurity, and $D^{(1)}$ and $D^{(2)}$ are the transmission probabilities proportional to the Tmatrix and to the T-matrix squared, respectively. The average current $j^{\sigma}$ can be calculated from Eq. (3) but with $D^{\sigma}\left(E, \rho-\rho_{0}, z\right)$ replaced with its average value

$$
<D^{\sigma}(E, z)>=\frac{N_{i m p}}{N} \int D^{\sigma}\left(E, \rho-\rho_{0}, z\right) d \rho,
$$

where the number of impurities $N_{i m p}=c N, c$ is the uniform impurity concentration, and $N$ is the total number of atoms in the plane. It is important to note that the average current is independent of $z$ satisfying the current continuity equation. In the following, we model an Fe/Oxide/Fe/Oxide/Fe DMTJ, with $V_{1}^{\uparrow}=V_{3}^{\uparrow}=V_{5}^{\uparrow}=$ $-4.58 \mathrm{eV}$ and $V_{1}^{\downarrow}=V_{3}^{\downarrow}=V_{5}^{\downarrow}=-0.66 \mathrm{eV}$ [4]. The barrier widths are $8 \AA$ Aand $2 \AA$, respectively, the impurity concentration is $c=5 \%$, and the impurity is placed at the center of the well.

In Fig. 2 we show the $T M R=\left(j_{P}-j_{A P}\right) / j_{A P}[6]$ as a function of the well thickness for the perfect DMTJ and in the presence of non-magnetic impurities with $V_{i m p}=0,+2,-2 \mathrm{eV}$. Here, $j_{P}\left(j_{A P}\right)$ is the current density in the parallel (antiparallel) configuration in which the leads are ferromagnetically (antiferromagnetically) aligned to the middle magnetic layer. The peak in the TMR at $\mathrm{b}=4.3 \AA$ for the perfect DMTJ can be dramatically enhanced (suppressed) by impurities with positive (negative) $V^{\sigma}$ which can shift the original QWS. For $\mathrm{b}=4.3 \AA$, the original QWS for the majority (minority) spin for the parallel (antiparallel) configuration are below $E_{F}$ and they are antisymmetric. On the other hand, 


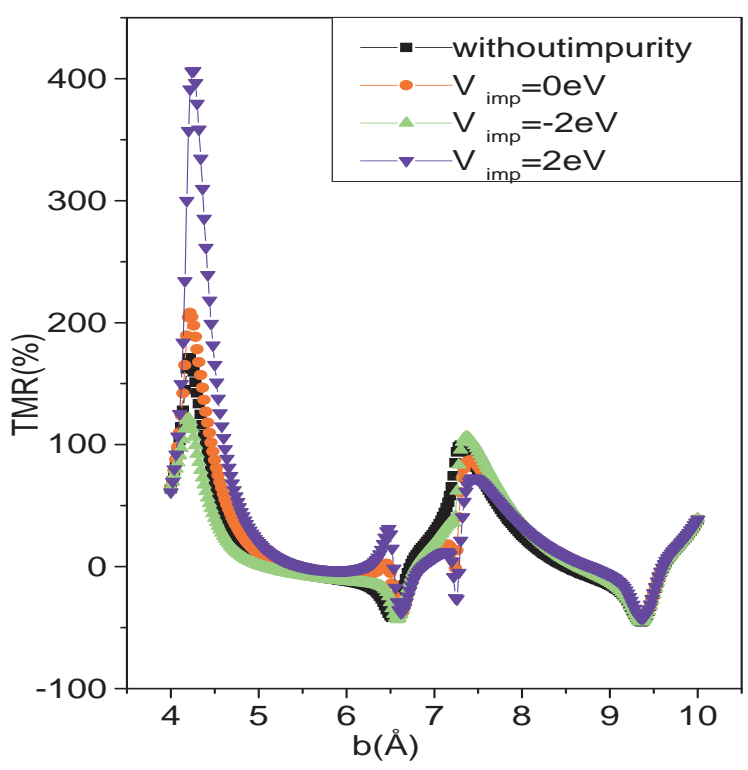

FIG. 2: TMR versus the middle layer thickness $b$ in the presence or absence of nonmagnetic impurities for different values of the impurity potential, $V_{i m p}$.

the QWS for the minority (majority) spin for the parallel (antiparallel) configuration are symmetric and above $E_{F}$. This is due to the larger (smaller) $k_{F}^{\sigma}$ in the well for the majority (minority) spin band [10]. The effect of the impurity on the QWS can be understood in terms of a simple quantum mechanical model, namely a $\delta$-function impurity-potential, $V^{\sigma}$, within a potential well. Symmetric QWS are shifted towards higher (lower) energies for positive (negative) impurity potential. In contrast, antisymmetric QWS are not shifted. Thus, the positive (negative) impurity-potential shifts the QWS away from (closer to) the Fermi energy, suppressing (enhancing) both $j_{P}^{\downarrow}$ and $j_{A P}^{\uparrow}$, and hence increasing (decreasing) the TMR. On the other hand, the $j_{P}^{\uparrow}$ and $j_{A P}^{\downarrow}$ are not affected by the impurity.

For $b=7.24 \AA$, in the absence of impurity the majority and minority spin QWS for both the parallel and antiparallel configurations are symmetric and below $E_{F}$. For $V_{i m p}=+2,0 \mathrm{eV}$, both the $V^{\uparrow}$ and $V^{\downarrow}$ are positive and the QWS are shifted closer to $\mathrm{E}_{F}$. Thus, the current for both parallel and antiparallel configurations increases and the TMR decreases. For $V_{i m p}=-2 \mathrm{eV}$ the QWS for the minority (majority) spin for the parallel (antiparallel) configuration are lowered in energy and hence the corresponding currents decrease. On the other hand, the QWS for the majority (minority) spin for the parallel (antiparallel) configuration are raised in energy and hence the corresponding currents increase. However, it turns out these current components compensate each other and the TMR is not altered much.

We next investigate the effect of a magnetic impurity on the spin polarization (SP) of the tunneling electrons in DMTJ. While the issue of SP for single barri-

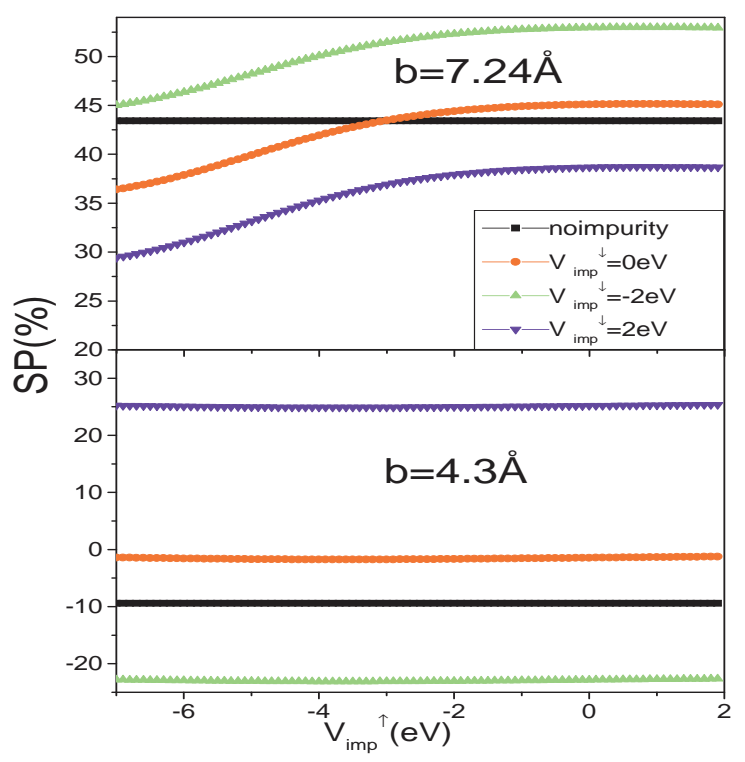

FIG. 3: Spin polarization for the parallel configuration as a function of $V_{i m p}^{\uparrow}$ in the presence or absence of impurities for different values of $V_{i m p}^{\downarrow}$ for $b=4.3 \stackrel{\AA}{A}$ and $7.24 \stackrel{\AA}{A}$, respectively.

ers has attracted significant interest recently, both theoretically [17, 18] and experimentally 2, 3], the SP in DMTJ in the presence or absence of disorder has not been addressed theoretically to the best of our knowledge. The SP for the parallel configuration is $S P=$ $\left(j_{P}^{\uparrow}-j_{P}^{\downarrow}\right) /\left(j_{P}^{\uparrow}+j_{P}^{\downarrow}\right)[2$, 3]. In Fig. 3 we show the SP for the parallel configuration as a function of $V_{i m p}^{\uparrow}$ for three values of $V_{i m p}^{\downarrow}$ for $b=4.3 \AA$ and $7.24 \AA$, respectively. For $\mathrm{b}=4.3 \AA$, the SP is independent of $V_{i m p}^{\uparrow}$ due to the fact that the QWS for the majority spin are antisymmetric and hence $j_{P}^{\uparrow}$ is not affected by the impurity. On the other hand, the SP depends on $V_{i m p}^{\downarrow}$ because the minority spin QWS are symmetric. In the absence of impurity, $j_{P}^{\uparrow}<j_{P}^{\downarrow}$ and hence the SP is negative. An impurity with $V^{\downarrow}>0$ decreases $j_{P}^{\downarrow}$ and hence increases the SP, leading to a sign reversal of the SP for large enough $V_{i m p}^{\downarrow}=2 \mathrm{eV}$. In contrast, $j_{P}^{\downarrow}$ increases if $V^{\downarrow}<0$ and hence the SP decreases.

For $\mathrm{b}=7.24 \AA$, the SP increases with increasing $V_{i m p}^{\uparrow}$ because the symmetric QWS below $\mathrm{E}_{F}$ are shifted close to the Fermi energy and $j_{P}^{\uparrow}$ increases. Note that for $V_{i m p}^{\uparrow}>-2 e V$ the SP varies weakly with $V_{i m p}^{\uparrow}$, due to the fact that the QWS lie in an energy range within $\mathrm{k}_{B} \mathrm{~T}$ around $E_{F}$. On the other hand, for $V_{i m p}^{\downarrow}=0$ and $2 \mathrm{eV}$, the QWS states are shifted closer to $E_{F}\left(V^{\downarrow}>0\right)$, and hence $j^{\downarrow}$ increases and the SP decreases. In contrast, for $V_{i m p}^{\downarrow}=-2 e V$ the QWS states are shifted away from $E_{F}$ $\left(V^{\downarrow}<0\right)$, and hence $j^{\downarrow}$ decreases and the SP increases.

In order to understand the effect of a non-magnetic impurity on the distribution of the local current in its 


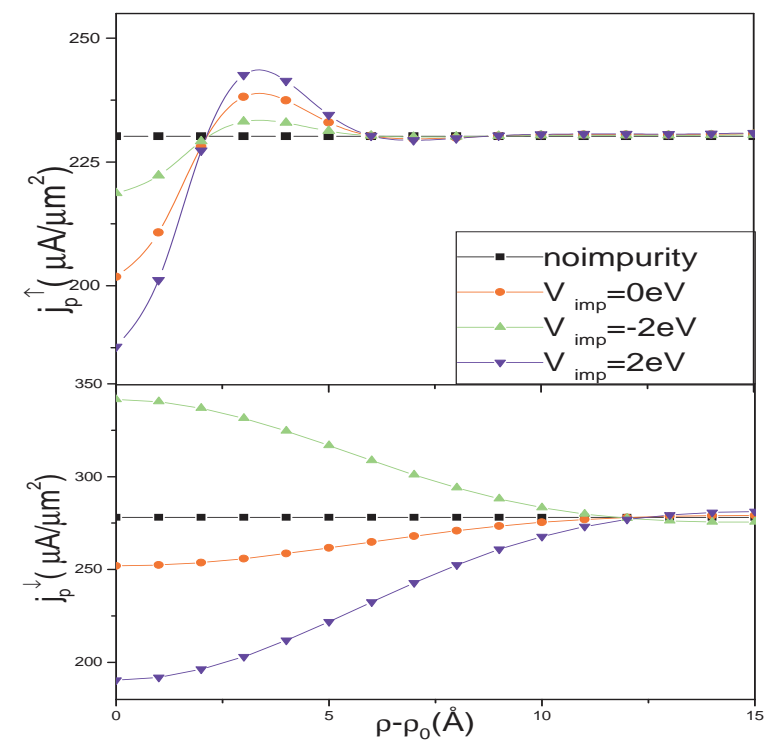

FIG. 4: Local current $j^{\sigma}\left(\rho-\rho_{0}\right)$ in the center of the first barrier as a function of $\rho-\rho_{0}$ for $b=4.3 \AA$ and for various values of $V_{i m p}$ for the parallel configuration. The nonmagnetic impurity is placed at the center of the well.

vicinity we present in Fig. 4 the local current, $j^{\sigma}\left(\rho-\rho_{0}\right)$, at the center of the first barrier as a function of $\rho-\rho_{0}$ for $b=4.3 \AA$ and for various values of $V_{i m p}$ for the parallel configuration. The non-magnetic impurity is placed at the center of the well. It is interesting to note that while the average current for the majority spin channel is not affected by the impurity due to the antisymmetric nature of the QWS (Fig. 2), the local current is dramatically changed compared to its corresponding value for the pure DMTJ. The effect is larger as the impurity potential increases. On the other hand, for the minority spin the local current in the vicinity of the impurity decreases for $V_{i m p}=0,+2 e V$ and increases for $V_{i m p}=-2 e V$, consistent with the behavior of the average current behavior (Fig. 1). For distances larger than $10 \AA$ the electrons do not feel the impurity.

In conclusion we have presented exact model calculations for the effect of impurities in the well on the spindependent resonant tunneling for DMTJ. To the best of our knowledge these are the first calculations to address the effect of impurity in DMTJ. The calculations reveal that the spin-dependent impurity scattering potential can tune selectively the majority and minority spin channels, giving rise to a wide variety of interesting and unusual transport phenomena. We find that the impurities can lead to a dramatic enhancement or suppression or sign reversal of the TMR and the spin polarization. The proposed underlying mechanism which explains consistently the overall behavior, is the shift of the original quantum well states depending on (i) the symmetric or asymmetric nature of these QWS and (ii) the impurity scattering potential. Our results for the local current indicate that even though the effect of the impurity on the average spin current is small for antisymmetric QWS, the local spin current exhibits strong variation.

We acknowledge useful discussions with J. Moodera. The research at California State University, Northridge was supported from NSF under Grant No DMR-00116566 and NASA under Grant No NCC5-513. A. Vedyayev is grateful to the Russian Foundation of Basic Research for financial support.
[1] Spin Electronics, ed. by M. J. Thornton and M. Ziese, Lecture Notes in Physics 569, (Springer Verlag, Berlin, 2001).

[2] J. S. Moodera, J. Nassar, and G. Mathon, Annu. Rev. Mater. Sci. 29, 381 (1999).

[3] E. Y. Tsymbal, O. N. Mryasov, and P. R. LeClair, J. Phys.: Condens. Matter. 15, R109 (2003).

[4] J. S. Moodera, J. Nowak, L. R. Kinder, P. M. Tedrow, R. J. M. van de Veerdonk, B. A. Smits, M. van Kampen, H. J. M. Swagten, and W. and J. M. de Jonge,Phys. Rev. Lett. 83, 3029 (1999).

[5] S. Yuasa, T. Nagahama, and Y. Suzuki, Science 297, 234 (2002).

[6] J. S. Moodera, L. R. Kinder, T. M. Wong, and R. Meservey, Phys. Rev. Lett. 74, 3273 (1995).

[7] A. G. Petukhov, A. N. Chantis, and D. O. Demchenko, Phys. Rev. Lett. 89, 107205 (2002).

[8] W. H. Butler, X.-G. Zhang, T. C. Schulthess, and J. M. MacLaren, Phys. Rev. B 63, 054416 (2001).

[9] M. Chshiev, D. Stoeffler, A. Vedyayev, and K. Ounadjela, Europhys. Lett. 58, 257 (2002).

[10] Z. Zheng, Y. Qi, D. Y. Xing, and J. Dong, Phys. Rev. B.
59, 14505 (1999).

[11] T. Hayashi, M. Tanaka, and A. Asamitsu J. Appl. Phys. 87, 4673 (2000).

[12] B. Wang, Y. Guo, and B. L. Gu, J. Appl. Phys. 91, 1318 (2002).

[13] X. Zhang, B. Z. Li, G. Sun, and F. C. Pu, Phys. Rev. B. 56, 5484 (1997).

[14] K. Miyamoto and H. Yamamoto, J. Appl. Phys. 84, 311 (1998).

[15] D. Fisher and P. Lee, Phys. Rev. B. 23, 6851 (1981).

[16] R. Jansen and J. S. Moodera, Phys. Rev. B 61, 9047 (2000).

[17] A. Vedyayev, D. Bagrets, A. Bagrets and B. Dieny, Phys. Rev. B. 63, 064429 (2001).

[18] E. Y. Tsymbal and D. G. Pettifor, Phys. Rev. B. 58, 432 (1998).

[19] C. B. Duke, Tunneling in Solids Solid State Physics 10, ed. by F. Seitz, D. Turnbull, and H. Ehrenreich, (Academic Press, New York, 1969).

[20] H. E. Camblong, P. M. Levy and S. Zhang, Phys. Rev. B 51, 16052 (1995). 